\title{
EVIDENCE OF THIN HELIUM ENVELOPES IN PG 1159 STARS
}

\author{
L. G. Althaus, ${ }^{1}$ A. H. Córsico, ${ }^{1}$ and M. M. Miller Bertolami ${ }^{1}$ \\ Facultad de Ciencias Astronómicas y Geofísicas, Universidad Nacional de La Plata, Paseo del Bosque s/n, (1900) La Plata, Argentina; \\ althaus@fcaglp.unlp.edu.ar, acorsico@fcaglp.unlp.edu.ar, mmiller@fcaglp.unlp.edu.ar \\ E. GarCía-BERRO ${ }^{2}$ \\ Departament de Física Aplicada, Escola Politècnica Superior de Castelldefels, Universitat Politècnica de Catalunya, \\ Avenida del Canal Olímpic, s/n, 08860 Castelldefels, Spain; garcia@fa.upc.edu \\ AND \\ S. O. KEPLER \\ Instituto de Física, Universidade Federal do Rio Grande do Sul, 91501-970 Porto Alegre, RS, Brazil; kepler@if.ufrgs.br \\ Received 2008 January 24; accepted 2008 February 21; published 2008 March 14
}

\begin{abstract}
We present evidence that PG 1159 stars could harbor He-rich envelopes substantially thinner than those predicted by current evolutionary models with current estimates of mass loss, which may be attributable to an extensive mass-loss episode during the born-again AGB phase. Specifically, we show that the models with thin He-rich envelopes predict remarkably large magnitudes of the rates of period change of the trapped and untrapped modes observed in the pulsating star PG 1159-035. This is a consequence of the much shorter evolutionary timescale of the models with thin He-rich envelopes during the low-gravity PG 1159 regime. Our findings are particularly interesting in view of the suggestion of an evolutionary link between the helium-deficient PG 1159 star H1504+65 and the recently discovered white dwarfs with almost pure carbon atmospheres.
\end{abstract}

Subject headings: dense matter — diffusion — stars: abundances — stars: evolution — stars: interiors — white dwarfs

Online material: color figure

\section{INTRODUCTION}

PG 1159 are hot and luminous stars that belong to the class of hydrogen-deficient post-asymptotic giant branch (AGB) stars. These stars are supposed to be the main progenitors of hydrogen-deficient white dwarfs, which account for $\sim 15 \%$ of the whole white dwarf population (Eisenstein et al. 2006). Currently, about 40 stars are members of the PG 1159 spectroscopic family (Werner \& Herwig 2006), which spans a wide range of surface gravities - $5.5 \leq \log g \leq 8$ (in cgs units) - and effective temperatures-75,000 K $\leqslant T_{\text {eff }} \leqslant 200,000 \mathrm{~K}$. PG 1159 stars are thought to be formed as a result of a born-again episode, that is, a very late thermal pulse (VLTP) experienced by a hot white dwarf during its early cooling phase-see Schönberner (1979) and Iben et al. (1983) for early references-or a late thermal pulse that occurs during the post-AGB evolution when $\mathrm{H}$ burning is still active (Blöcker 2001). During the VLTP, the helium flash-driven convection zone reaches the hydrogenrich envelope of the star and, consequently, most of the hydrogen is burned. The star is then forced to evolve rapidly back to the AGB and finally as the central star of a planetary nebula at high $T_{\text {eff }}$ values. A striking feature characterizing PG 1159 stars is their peculiar surface chemical composition. Spectroscopic analyses have revealed that most PG 1159 stars exhibit helium-, carbon-, and oxygen-rich surface abundances. The typical surface mass abundances of PG 1159 stars are $X_{\mathrm{He}} \simeq$ $0.33, X_{\mathrm{C}} \simeq 0.5$, and $X_{\mathrm{O}} \simeq 0.17$, although notable variations are found from star to star (Dreizler \& Heber 1998; Werner 2001). The variety of surface patterns observed in PG 1159 stars poses indeed a challenge to the theory of stellar evolution. In par-

\footnotetext{
${ }^{1}$ Member of the Carrera del Investigador Científico y Tecnológico, CONICET (IALP), Argentina.

${ }^{2}$ Institut d'Estudis Espacials de Catalunya, Calle Gran Capità 2-4, 08034 Barcelona, Spain.
}

ticular, the appreciable abundance of oxygen in the atmospheres of these stars has been successfully explained by Herwig et al. (1999) on the basis of evolutionary calculations of the bornagain scenario that incorporate convective overshoot.

Interest in PG 1159 stars is also motivated by the fact that more than 10 of them, the so-called DOVs, exhibit multiperiodic luminosity variations, attributable to global nonradial $g$ mode pulsations, a fact that has attracted much attention-see, for instance, Gautschy et al. (2005) and Córsico et al. (2006). Recently, considerable observational effort has been devoted to the study of some pulsating PG 1159 stars. In particular, Vauclair et al. (2002) have presented asteroseismological results for RX J2117.1+3412 on the basis of a multisite photometric campaign. More recently, Fu et al. (2007) have detected a total of 23 frequencies in PG $0122+200$ and Costa et al. (2008) have enlarged to 198 the total number of pulsation modes in PG 1159-035-the prototype of the class-making it the star with the largest number of modes detected besides the Sun.

Parallel to these observational breakthroughs, substantial progress in the theoretical modeling of PG 1159 stars has been possible (Herwig et al. 1999; Althaus et al. 2005; Lawlor \& MacDonald 2006). In this sense, the full set of PG 1159 evolutionary models developed by Miller Bertolami \& Althaus (2006) has proved to be valuable for deriving structural parameters of pulsating PG 1159 on the basis of individual period fits-see Córsico et al. (2007a) and (2007b) respectively for an application to the hot pulsating star RX J2117.1+3412 and to the coolest member of the class, PG $0122+200$. These evolutionary models are derived from the complete evolutionary history of progenitor stars with different stellar masses and an elaborate treatment of the mixing and extra-mixing processes during the violent hydrogen burning episode that occurs during the born-again phase. The success of these models at explaining both the spread in the surface chemical compositions observed 
in PG 1159 stars and the location of the DOV instability strip in the $g-T_{\text {eff }}$ plane (Córsico et al. 2006) gives reliability to the inferences drawn from individual pulsating PG 1159 stars.

However, except for PG $0122+200$, the stellar masses derived from individual period fits differ considerably from the masses obtained using spectroscopic data-although the spectroscopic uncertainty $(\Delta \log g \simeq 0.5$ ) is large. This is noteworthy in the case of RX J2117.1+3412, for which the asteroseismological mass is about $25 \%$ smaller than the spectroscopic value (Corsico et al. 2007a). This discrepancy could be indicative of a missing piece of physics in the existing PG 1159 evolutionary models, a suspicion that becomes more solid in light of the detailed seismological study of the pulsating star PG 1159-035 recently presented by Córsico et al. (2008). Indeed, the best-fit model derived by these authors for PG $1159-035$ is located outside the predicted dipole instability strip. Also, the best-fit model fails to predict a mixture of positive and negative rates of change of the periods ( $\Pi$ ) as recently reported by Costa \& Kepler (2008) in PG 1159-035. But most importantly, the best-fit model and the evolutionary models of Miller Bertolami \& Althaus (2006) - and of other authors as well — do not predict the large magnitude of the rates of period change observed in PG 1159-035 (Costa et al. 1999). This is a well-known shortcoming - see Kawaler \& Bradley (1994) and Córsico et al. (2008)—of the standard evolutionary models of PG 1159 stars which predict theoretical $\Pi$ values about 1 order of magnitude smaller than those observationally derived.

We present here solid evidence that PG 1159 stars could be characterized by substantially thinner helium-rich envelopes than traditionally accepted from the standard theory of the formation and evolution of PG 1159 stars (Herwig et al. 1999; Miller Bertolami \& Althaus 2006), which predicts that postborn-again remnants are expected to retain thick He envelopes. For instance, the mass of the He envelope for the $0.55 M_{\odot}$ sequence of Miller Bertolami \& Althaus (2006) after emerging from the born-again episode amounts to $0.03 M_{\odot}$. The possibility that PG 1159 stars could harbor thinner He envelopes is sustained by the fact that V4334 Sgr (Sakurai's object), a star emerging from a born-again episode, is displaying very strong mass-loss episodes as it is rapidly reheating (van Hoof et al. 2007). In this Letter we compute full evolutionary sequences for post-born-again stars under the assumption that such mass-loss episodes reduce the mass of the He envelope considerably. We find that PG 1159 evolutionary sequences with thin He envelopes evolve much faster than the standard ones. On the basis of detailed pulsation calculations, we find that PG 1159 models with thin He envelopes predict rates of period change which are in excellent agreement with observations in the case of PG 1159-035 (Costa \& Kepler 2008), thus solving the long-standing discrepancy between the observed and theoretical rate of period change in this star.

\section{EVOLUTIONARY SEQUENCES}

For the purpose of this Letter we have calculated full evolutionary sequences appropriate for PG 1159 stars that were evolved directly from the born-again phase. The calculations have been done with the LPCODE evolutionary code that computes the formation and evolution of white dwarf stars through late thermal pulses (Althaus et al. 2005) on the basis of a detailed description of the physical processes involved in the calculation of the violent hydrogen-burning event during the born-again stage, particularly, diffusive overshooting and non- instantaneous mixing. This code has been employed to derive a full set of PG 1159 evolutionary sequences starting from ZAMS progenitors with initial mass ranging from 1.0 to $3.75 M_{\odot}$ (Miller Bertolami \& Althaus 2006). All of the sequences have been followed from the ZAMS through the thermally pulsing phase on the AGB and finally to the born-again stage where the remaining hydrogen of the remnants is burned.

As recently discussed in Miller Bertolami \& Althaus (2007) the born-again models of Miller Bertolami \& Althaus (2006) hint at a consistent picture with the observed behavior of Sakurai's object, particularly its extremely fast post-VLTP evolution. In addition, very strong mass-loss episodes during the Sakurai stage appears to be a necessary ingredient in the evolutionary calculations (Miller Bertolami \& Althaus 2008) to bring the evolutionary timescales into agreement with the short reheating timescale of the Sakurai's object after its maximum brightness reported by van Hoof (2007). Remarkably, strong mass-loss episodes have recently been reported by van Hoof et al. (2007), who suggested that the mass ejected by Sakurai's object in the VLTP could be as high as $10^{-2} M_{\odot}$. In this Letter, we concentrate on the consequences of such mass-loss events for the subsequent evolution of the born-again remnants during the PG 1159 stage. To this end, we have considered a rather extreme situation, but still reasonably consistent with the results of van Hoof et al. (2007), in which a large fraction of the remaining He envelope is lost as a consequence of mass-loss episodes once the hydrogen-deficient star is back to the AGB. Specifically, we have concentrated on the $0.556 M_{\odot}$ post-VLTP sequence of Miller Bertolami \& Althaus (2007), which after the VLTP is characterized by a He envelope of $0.032 M_{\odot}$. We assume that the star loses $\sim 0.02 M_{\odot}$ as a result of mass-loss episodes. Hereafter, this sequence will be referred to as the thin-He-envelope sequence. This allows the study PG 1159 evolutionary model sequences with structurally different envelopes from those predicted by the standard theory of stellar evolution for post-born-again stars.

\section{RESULTS AND IMPLICATIONS}

The most important consequence of the strong envelope reduction caused by mass-loss episodes during the Sakurai stage is that the helium shell burning becomes virtually extinct. The remnant is left with no available nuclear sources and, as a result, its evolution through the low-gravity PG 1159 regime proceeds on a much shorter timescale than in the standard PG 1159 sequences in which helium burning mostly contributes to the surface luminosity of the models. The marked reduction in the evolutionary timescales characterizing the models with thin $\mathrm{He}$ envelopes is expected to yield large rates of period change in low-gravity pulsating. PG 1159 stars, which is a feature needed to explain the large $\Pi$ measured in PG $1159-035$ itself. For a quantitative inference of the possible impact, we have computed $l=1 g$-mode adiabatic pulsation periods for our PG 1159 models using the pulsation code described in Córsico \& Althaus (2006). In particular, we concentrate on models belonging to our sequences with thin and thick He envelopes characterized by effective temperatures and surface gravities around the spectroscopically determined values for $\mathrm{PG}$ $1159-035$.

In Figure 1, which summarizes the main result of this work, we show the rate of period change $\Pi$ for periods in the range $300 \mathrm{~s} \leq \Pi \leq 1000 \mathrm{~s}$. The results corresponding to PG 1159 models with thin (thick) He envelopes are shown with solid (dashed) lines and symbols. The model with the thin He en- 


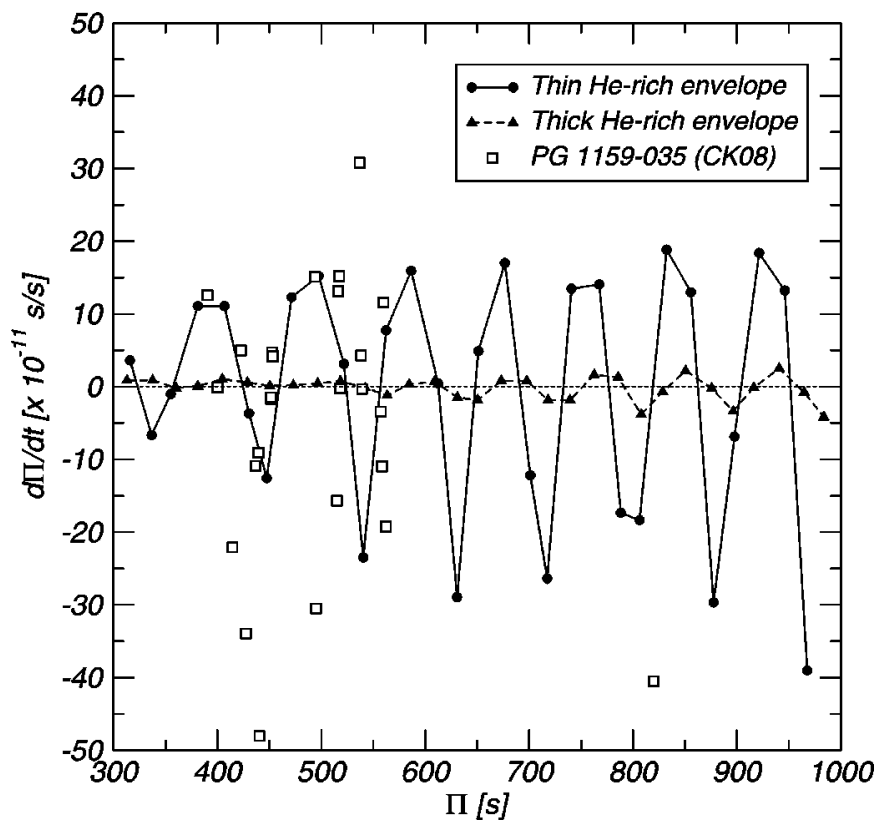

FIG. 1.- Rate of period change for our PG 1159 models. The solid (dashed) line and symbols correspond to the model characterized by a thin (thick) $\mathrm{He}$ envelope. The open squares display the observed rates of period change in PG 1159-035 according to Costa \& Kepler (2008). Note that the model with a thin $\mathrm{He}$ envelope predicts the remarkably large variations of the rates of period change of the trapped and untrapped modes observed in this star.

velope is characterized by a stellar mass of $0.534 M_{\odot}$, a He envelope of $0.012 M_{\odot}, T_{\text {eff }} \sim 160,000 \mathrm{~K}, \log L / L_{\odot} \sim 2.88$, and $\log g \sim 7.1$. In both models, modes trapped in the outer envelope are characterized by negative rates of period change. This figure demonstrates that the values of $\dot{\Pi}$ of the model with a thin $\mathrm{He}$ envelope far exceed those predicted by the model with a thick He envelope. This is a consequence of the much shorter evolutionary timescale of the model with a thin $\mathrm{He}$ envelope during the low-gravity PG 1159 regime. Indeed, for untrapped modes $(\dot{\Pi}>0)$ the rates can be up to an order of magnitude larger in the case in which a thin He envelope is adopted, and even larger for trapped modes $(\dot{\Pi}<0)$. On the contrary, for the regime of the evolved pulsating PG 1159 stars of high gravity such as PG $0122+200$, already on the hot white dwarf cooling track, where helium shell burning is no longer the luminosity source in the standard models, the magnitude of the period changes becomes much less sensitive to the thickness of the helium envelope, as noted earlier by Kawaler \& Bradley (1994). In Figure 1 we also include the observed rates of period change in PG 1159-035 (Costa \& Kepler 2008), which are displayed as open squares. Note that PG 1159-035 exhibits a mixture of positive and negative rates of large amplitude (up to $\approx 40 \times 10^{-11} \mathrm{~s} \mathrm{~s}^{-1}$ ). The general agreement between the $\Pi$ values predicted by the model with a thin $\mathrm{He}$ rich envelope and those observed in PG 1159-035 is remarkable. Note in particular that the rate of period change, $\dot{\Pi}=$ $(+15.17 \pm 0.045) \times 10^{-11} \mathrm{~s} \mathrm{~s}^{-1}$, of the $517.1 \mathrm{~s}$ mode- the central peak of one of the PG 1159-035 highest amplitude modes-is well reproduced by the model with a thin He-rich envelope, in sharp contrast with the predictions of the standard model with a thick envelope, for which the values of $\Pi$ remain smaller than $2 \times 10^{-11} \mathrm{~s} \mathrm{~s}^{-1}$ for the relevant range of periods. From these results, it is clear that the presence of a thin Herich envelope in PG 1159-035 appears to be a key ingredient to resolve the long-standing order-of-magnitude discrepancy

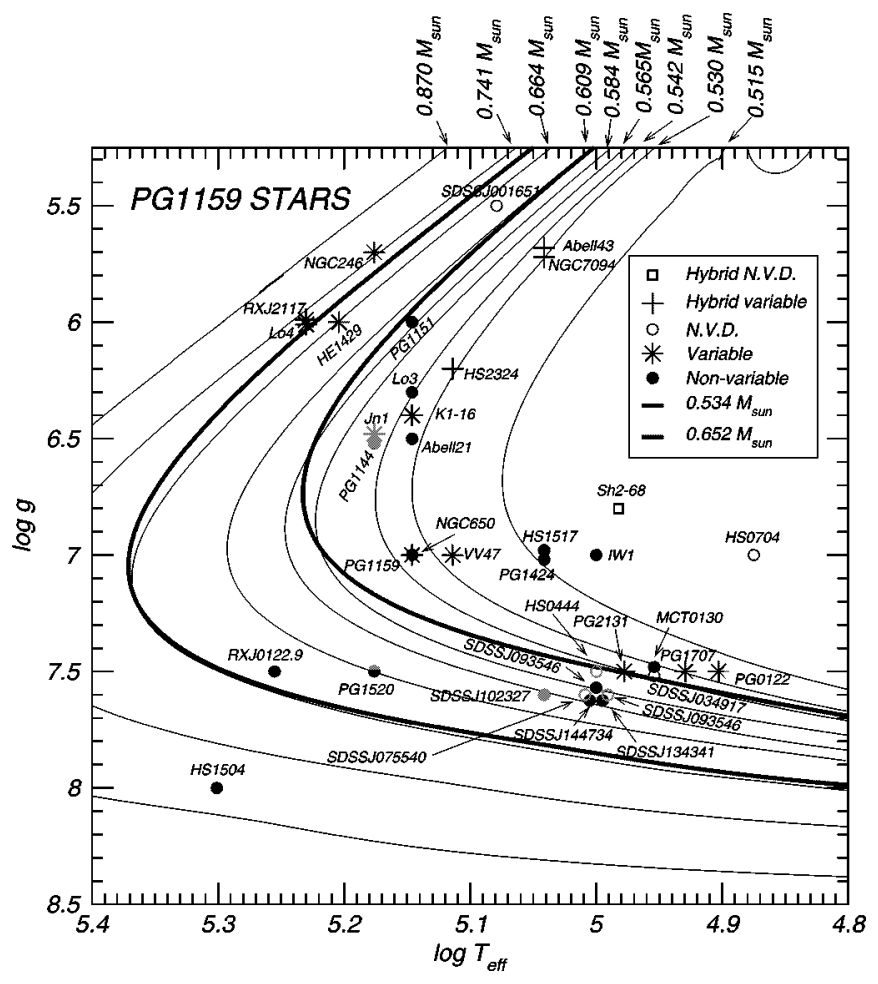

FIG. 2.-Thick He-layer evolutionary sequences of Miller Bertolami \& Althaus (2006) in the $g-T_{\text {eff }}$ plane for different stellar masses (thin lines) and the 0.535 and $0.652 M_{\odot}$ PG 1159 model sequences characterized by thin He-rich envelopes described in this Letter (thick lines). Note that these sequences have substantially smaller surface gravities than their thick He-rich counterparts, particularly for the low-gravity regime. The available observational data for selected PG 1159 stars are also included. Spectroscopic uncertainties are within 0.5 dex for the gravity and between $10 \%$ and $15 \%$ for the effective temperature. [See the electronic edition of the Journal for a color version of this figure.]

between the predictions of standard PG 1159 evolutionary models and the observed rates of period change in $P G$ 1159-035. We want to mention that the effective temperature of our model with thin He envelope is somewhat larger than the spectroscopically inferred value for PG 1159-035. However, this inconsistency does not affect the main conclusion of this Letter about the strong impact of thin He envelopes on the predicted rate of period changes in this pulsating PG 1159 star. More precise asteroseismological fits to the observed period and period changes in PG 1159-035 with sequences with thin He envelopes would require the calculation of a much finer grid of evolutionary sequences, an issue that would carry us beyond the scope of the present Letter.

Another implication of PG 1159 stars having thin He-rich envelopes concerns their location in the $g-T_{\text {eff }}$ plane. In fact, the evolutionary tracks in this plane are strongly modified if the mass of the He-rich envelope is reduced. This can be seen in Figure 2 where the standard evolutionary sequences of Miller Bertolami \& Althaus (2006) - thin lines - are shown together with two sequences of masses 0.535 and $0.652 M_{\odot}$ computed with thin He-rich envelopes (solid lines), characterized by envelopes of 0.012 and $0.008 M_{\odot}$, respectively-as compared with the envelope mass of the standard models of 0.032 and $0.02 M_{\odot}$. Note that for the low-gravity regime, tracks characterized by thin He-rich envelopes are hotter than their thick He-rich counterparts. That is, at a given effective temperature, thin He-rich sequences are characterized by markedly smaller gravities than the sequences with thick envelopes. This will certainly affect the spectroscopic mass determinations. This is 
a relevant issue concerning the hottest known pulsating $\mathrm{PG}$ 1159 star, RX J2117.1+3412, a low-gravity PG 1159 star for which the spectroscopic mass-about $0.72 M_{\odot}$ (Miller Bertolami \& Althaus 2006) - is much larger than the seismological mass of $0.56 M_{\odot}$ (Vauclair et al. 2002; Córsico et al. 2007a). Note that the models with thin envelopes predict for $\mathrm{RX}$ $\mathrm{J} 2117.1+3412$ a spectroscopic mass of about $0.65 M_{\odot}$, considerably smaller than that derived from thick He-rich models. Hence, PG 1159 sequences with thin He-rich envelopes may help to alleviate the discrepancy between the spectroscopic and seismic mass of RX J2117.1+3412.

\section{CONCLUSIONS}

In this Letter we have presented evidence that PG 1159 stars-or at least PG 1159-035 itself-could harbor He-rich envelopes thinner than those predicted previously. The existence of PG 1159 stars with thin He-rich envelopes is in line with the reported strong mass-loss episodes in Sakurai's object (van Hoof et el. 2007), an emerging post-born-again star that is expected to evolve to the PG 1159 domain. Specifically, we have shown that if the PG 1159 progenitors emerge from their born-again episode with considerably reduced envelopes, then the predicted rates of period change in low-gravity pulsating PG 1159 stars are profoundly altered to such an extent that the long-standing discrepancy with the rate of period change measured in PG 1159-035 is solved. This result opens the possibility that PG 1159 stars in general could be characterized by thinner He-rich envelopes than traditionally accepted on the basis of the standard theory of post-AGB evolution. Pushing our results to their limits, we are tempted to speculate that the helium deficiency observed in $\mathrm{H} 1504+65$, the most massive known PG 1159 star, could be the result of stronger mass-loss episodes than those observed in Sakurai's object, which combined with the smaller helium content that remains in massive post-VLTP stars, could lead to PG 1159 remnants almost devoid of helium. The existence of PG 1159 stars with thin helium envelopes is particularly interesting in view of the suggestion of an evolutionary connection between the helium-deficient PG 1159 star H1504+65 and the recently discovered white dwarf population with carbon atmospheres (Dufour et al. 2007).

Part of this work was supported by PIP 6521 grant from CONICET, by MEC grant AYA05-08013-C03-01, by the European Union FEDER funds, and by the AGAUR.

\section{REFERENCES}

Althaus, L. G., Serenelli, A. M., Panei, J. A., Córsico, A. H., García-Berro,

E., \& Scóccola, C. G. 2005, A\&A, 435, 631

Blöcker, T. 2001, Ap\&SS, 275, 1

Córsico, A. H., \& Althaus, L. G. 2006, A\&A, 454, 863

Córsico, A. H., Althaus, L. G., Kepler, S. O., Costa, J. E. S., \& Miller Bertolami, M. M. 2008, A\&A, 478, 869

Córsico, A. H., Althaus, L. G., \& Miller Bertolami, M. M. 2006, A\&A, 458, 259

Córsico, A. H., Althaus, L. G., Miller Bertolami, M. M., \& Werner, K. 2007a, A\&A, 461, 1095

Córsico, A. H., Miller Bertolami, M. M., Althaus, L. G., Vauclair, G., \& Werner, K. $2007 \mathrm{~b}$, A\&A, 475, 619

Costa, J. E. S., \& Kepler, S. O. 2008, A\&A, in press

Costa, J. E. S., Kepler, S. O., \& Winget, D. E. 1999, ApJ, 522, 973

Costa, J. E. S., et al. 2008, A\&A, 477, 627

Dreizler, S., \& Heber, U. 1998, A\&A, 334, 618

Dufour, P., Liebert, J., Fontaine, G., \& Behara, N. 2007, Nature, 450, 522
Eisenstein, D. J., et al. 2006, ApJS, 167, 40

Fu, J. N., et al. 2007, A\&A, 467, 237

Gautschy, A., Althaus, L. G., \& Saio, H. 2005, A\&A, 438, 1013

Herwig, F., Blöcker, T., Langer, N., \& Driebe, T. 1999, A\&A, 349, L5

Iben, I., Kaler, J. B., Truran, J. W., \& Renzini, A. 1983, ApJ, 264, 605

Kawaler, S. D., \& Bradley, P. A. 1994, ApJ, 427, 415

Lawlor, T. M., \& MacDonald, J. 2006, MNRAS, 371, 263

Miller Bertolami, M. M., \& Althaus, L. G. 2006, A\&A, 454, 845 2007, MNRAS, 380, 763 2008, in ASP Conf. Ser., Hydrogen-Deficient Stars, ed. K. Werner \&

T. Rauch (San Francisco: ASP), in press (arXiv:0711.4565v1)

Schönberner, D. 1979, A\&A, 79, 108

van Hoof, P. A. M., et al. 2007, A\&A, 471, L9

Vauclair, G., et al. 2002, A\&A, 381, 122

Werner, K. 2001, Ap\&SS, 275, 27

Werner, K., \& Herwig, F. 2006, PASP, 118, 183 